\title{
UTILIZACIÓN DEL AUDIOBLOG COMO INSTRUMENTO FORMATIVO Y GENERADOR DE APRENDIZAJE EN EL AULA
}

David Hortigüela Alcalá ${ }^{1}:$ Universidad de Burgos. España

dhortiguela@ubu.es

Ángel Pérez Pueyo: Universidad de León. España angel.perez.pueyo@unileon.es

\section{RESUMEN}

El presente estudio, realizado en la Facultad de Educación de Burgos en el curso escolar 2013-2014, analiza la percepción del alumnado universitario sobre la motivación y la relación social en el aula que genera el uso del audioblog como elemento formativo y de aprendizaje. Participan 172 alumnos de 4 asignaturas de dos titulaciones (Primaria e Infantil), así como los dos docentes que imparten dichas asignaturas. Se generan dos grupos, grupo A (uso del audioblog) y grupo B (no uso del audioblog). La metodología de la investigación ha sido mixta, empleándose tanto un análisis cuantitativo (descriptivo e inferencial) como cualitativo (entrevistas). Se emplea un pretest-postest, comprobando en qué medida varía la motivación del alumnado y el clima social del aula tras haber cursado las asignaturas. Se observa cómo los estudiantes que han utilizado el audioblog como elemento formativo presentan una mejora en el clima social de clase tanto en relación al pretest como respecto al grupo que no lo ha utilizado. La edad en el grupo A y la asiduidad en el uso de las nuevas tecnologías (NNTT) en el grupo B son las variables que presentan diferencias entre grupos en relación al aprendizaje generado en la materia. Por otra parte, los docentes reflejan disparidad de opiniones respecto al uso formativo que pueden tener las NNTT en el aula.

PALABRAS CLAVE: audioblog - nuevas tecnologías - motivación - relación social percepción docente - trabajo en grupo - metodología

${ }^{1}$ David Hortigüela Alcalá: Doctor Internacional en Educación. Máster en Educación y Sociedades Inclusivas. Licenciado en Ciencias de la Actividad Física y del Deporte.

Correo: dhortiguela@ubu.es 


\section{THE USE OF AUDIOBLOG AS A FORMATIVE ELEMENT AND GENERATOR FOR LEARNING IN THE CLASSROOM.}

\section{ABSTRACT}

This study, conducted at the Faculty of Education of Burgos in the school year 20132014, analyzes the perception of university students on motivation and social relationships in the classroom that generates audioblog use as a training element and learning. In the study 172 students in 4 subjects of two degrees (Primary and Child) and the two teachers who teach the subjects are involved. Two groups, group A (use audioblog) and group B (no use of audioblog) are generated. The research methodology has been mixed, using both a quantitative analysis (descriptive and inferential) and qualitative (interviews). A pretest-posttest is used, checking how varies student motivation and social climate of the classroom after having taken the subjects. It shows how students who have used audioblog as a training element present an improvement in the social climate in class than those who didn't use it. The age in group A and prior experience in the use of new technologies (NT) in group B are the variables that differ between groups in relation to learning generated in the subject. Moreover, teachers reflect a difference of opinion regarding the formative use may have NT in the classroom.

KEY WORDS: audioblog - new technologies - motivation - social relationship teacher perception - workgroup - methodology.

\section{INTRODUCCIÓN}

El uso de las NNTT en la sociedad actual es un hecho que impregna las actividades cotidianas, habiéndose convertido en algo rutinario que se realiza de manera inconsciente (Davis, 2011). Atendiendo al ámbito educativo, las posibilidades existentes son infinitas, estando disponibles de manera accesible y gratuita una gran cantidad de herramientas y recursos aplicables en diversidad de contextos y situaciones (Martínez, 2013). Este uso puede llevarse a cabo en todas las etapas educativas, desde la de Infantil, mediante un uso más intuitivo y de familiarización, hasta el universitario, donde el alumno ha de demostrar su uso autónomo y aplicable a la realidad social (Patrut, Patrut \& Cmeciu, 2013). Definir el término de NNTT no es algo sencillo, pero en relación a la educación ha de enfocarse como el uso de las herramientas que favorecen los procesos de comunicación entre las personas que las utilizan de manera interactiva, posibilitando el aprendizaje de los usuarios y la motivación por indagar y motivarse hacia las temáticas planteadas (Brodahl, Hadjerrouit, \& Hansen, 2011). No obstante Sáez-López (2012) destaca que su principal uso en el aula no habrá de estar relacionado únicamente con su dominio y manejo, sino con la manera en la que pueden ser utilizadas para alcanzar los objetivos 
propios de cada materia.

Estrechamente relacionado con el ámbito comunicativo, en esta investigación se ha utilizado el audioblog, entendido como una herramienta interactiva que facilita la manera de compartir información generada de manera autónoma por el usuario (Hsu, Wang, Comac, 2008). En un audioblog se presentan diferentes ficheros audios publicados regularmente por uno o varios estudiantes. La riqueza del mismo se encuentra en la retroalimentación existente entre los alumnos. Tan y Tan (2010) indican que el audioblog es un paso más del blog tradicional, ya que permite que los estudiantes suban sus propias producciones de manera oral, algo que va más allá de insertar un link, vídeo, imagen de una web o hacer un retweet. Además evita el problema del filtro (que siempre es preciso hacer) sobre la información que se extrae de internet, ya que el archivo es generado únicamente por el alumno de manera autónoma, otorgando así un carácter personal a su producción (Wang, Ke, Wu, \& Hsu, 2012). Del mismo modo se potencia la exposición oral, destreza fundamental para el futuro maestro. Es interesante observar cómo el alumno analiza una información para después elaborar un discurso de manera sintetizada que será grabado en audio, obteniendo con posterioridad un feedback con los compañeros.

Sin embargo, como establece Meneses y Llorente (2010) es fundamental otorgar un carácter formativo a este tipo de herramientas, ya que si no su uso puede conllevar efectos no tan positivos como la mera subida de archivos que no se relacionen con la temática abordada en el aula y que no se asocien a una retroalimentación efectiva por parte de los compañeros y el docente. El uso de las plataformas virtuales que permiten la interacción entre todos sus participantes, como pueden ser los blogs, han de estar reguladas por una serie de principios de actuación en los que se aclare: asiduidad en las intervenciones, calidad de las mismas, vinculación con lo trabajado en el aula y aportaciones a lo comentado por los compañeros (Gerich, 2013). Esto favorecerá su pleno uso formativo y evitará una utilización del mismo alejada de los fines educativos. Como establecen Salmerón, Rodríguez y Gutiérrez (2011), un docente cuando implanta una herramienta virtual de este tipo ha de ser consciente del elemento evaluador que supone, por lo que debe registrar la información que le proporciona el mismo así como la funcionalidad para la adquisición de los contenidos y competencias de la asignatura.

En relación a la presentación oral de trabajos por parte del alumno, (Ryan, 2013) establece que desde la implantación de Bolonia éstos han aumentado sustancialmente, lo que no indica que su calidad también sea mayor. En este sentido, sería necesario valorar de manera reflexiva qué es lo que pretende el docente cuando demanda exposiciones orales sobre trabajos en grupo y cuáles son las competencias que se alcanzan, porque... ¿Verdaderamente es formativo que cada integrante del grupo se aprende su parte de la exposición y no conozca la del resto de compañeros? ¿No se abusa demasiado de exposiciones de trabajos sin que se le dedique el tiempo suficiente a la preparación de los mismos? ¿No hay otras maneras de que el alumno comparta sus experiencias con los compañeros que no sea mediante la presentación de una serie de diapositivas? Y quizás lo más importante, ¿de qué manera el alumno se motiva más hacia la temática abordada? En función de estas preguntas surge la 
presente investigación, comprobando en qué medida la utilización de estas herramientas (en este caso el audioblog) pueden provocar un mayor nivel de aprendizaje y de relación social en el aula.

Por lo tanto, es fundamental que el docente reflexione sobre la relación existente entre los procedimientos de evaluación planteados en la asignatura y los aprendizajes que se pretenden conseguir con los mismos, adaptando éstos a los instrumentos utilizados (Nortes \& Nortes-Checa, 2012). En palabras de White (2007), si como profesores pretendemos que el alumno alcance una autonomía y conocimiento elevado sobre los contenidos de las asignaturas impartidas, es preciso que exista un ajuste entre las pretensiones del docente y discente. Este hecho es lo que diferenciará un uso lógico y consensuado de uno impuesto y unidireccional (Vera, 2010). Experiencias como la de Leemen (2013) indican que uno de los principales factores que favorece la implicación del alumnado en las asignaturas es la generación de un clima positivo entre los integrantes del aula. Para ello, la implantación de instrumentos formativos vinculados a las NNTT puede convertirse en un medio idóneo para descubrir diferentes vías de aprendizaje. Lo que tiene que quedar claro es la retroalimentación necesaria entre los participantes del audioblog, analizando el tipo de archivos que se cuelgan, la información que en ellos se plantea, las aportaciones de los compañeros y la vinculación de esa información con otra del temario o de la web en la que se pueda profundizar.

En esta investigación se analizarán las variables de motivación y clima social en el aula mediante un pretest-postest y contraste entre grupos a partir de un instrumento formativo (audioblog), algo sobre lo que la bibliografía actual no incide demasiado.

\section{OBJETIVOS}

1. Comparar la percepción del alumnado sobre su motivación hacia las tareas y la relación social generada en el aula entre los grupos que han utilizado el audioblog como elemento formativo y aquellos que no.

2. Analizar el grado de influencia que tiene las variables de edad, experiencia previa en el uso de las NNTT y número de veces que han trabajado en grupo a lo largo de la carrera en el nivel de aprendizaje obtenido por el alumno al finalizar las asignaturas.

3. Contrastar las opiniones que tienen los docentes sobre la utilización de las NNTT como herramienta óptima de fomento de motivación en el alumno.

\section{METODOLOGÍA}

\subsection{Participantes}

Para este estudio han participado de 162 alumnos (63,7\% mujeres y $36,3 \%$ hombres), con una media de edad de 21.53 años (DT $=2.41$ ). Toda los estudiantes que ha cumplimentado el cuestionario forman parte de 4 asignaturas impartidas en el cuso escolar 2013-2014 en la Facultad de Educación de Burgos. Pertenecen a 2 
grados: Primaria (grupo A, 75 alumnos) e Infantil (grupo B, 87 alumnos). El grupo A ha utilizado en las dos asignaturas de Primaria el audioblog como elemento formativo, mientras que el B No. Las dos asignaturas del grupo A son impartidas por un docente y las dos del grupo B por otro. Tras realizar la prueba de Shapiro-Wilk se observa que la muestra responde a parámetros de normalidad $(p=.131)$.

\begin{tabular}{ccccc}
\hline ASIGNAURA & GRADO & CURSO & $\begin{array}{c}\text { USO } \\
\text { AUDIOBLOG }\end{array}$ & No DE ALUMNOS \\
\hline $\begin{array}{c}\text { Educación } \\
\text { Física y su } \\
\text { Didáctica }\end{array}$ & Primaria & 20 & SÍ (GRUPO A) & 40 \\
\hline $\begin{array}{c}\text { Juego } \\
\text { Educativo }\end{array}$ & Primaria & 40 & SÍ (GRUPO A) & 35 \\
\hline $\begin{array}{c}\text { Desarrollo } \\
\text { Psicomotor I }\end{array}$ & Infantil & 20 & NO (GRUPO B) & 41 \\
\hline $\begin{array}{c}\text { Desarrollo } \\
\text { Psicomotor } \\
\text { II }\end{array}$ & Infantil & 40 & NO (GRUPO B) & 46 \\
\hline & & & TOTAL ALUMNOS & $\mathbf{1 6 2}$ \\
\hline
\end{tabular}

Tabla 1. Participantes en el estudio (asignatura, grado, uso de audioblog y número de participantes en cada asignatura.

\subsection{Instrumentos}

\subsubsection{Cuantitativo}

Como instrumento de recogida de datos se ha utilizado el cuestionario validado de evaluación en formación inicial (Castejón, Santos y Palacios, 2013). Para ello, y con el fin de obtener la garantía en la percepción del alumnado sobre su motivación hacia la asignatura y el clima relacional alcanzado en el aula, el estudiante cumplimentó específicamente la Escala de Sistemas de Evaluación en Formación Inicial del Profesorado. Esta escala tiene un total de 21 cuestiones a las que los discentes responden en grado al acuerdo con el enunciado en una escala tipo Likert, cuyos valores van desde 1 (Nada) hasta 5 (Mucho). El cuestionario presenta una fiabilidad del Alpha de Cronbach de 0,831, superior al límite inferior que según Corbetta (2007) es aceptado como fiable. Se aplica un nivel de confianza del 95\%. Además, esta escala se construye a partir de la literatura especializada en el ámbito universitario, siendo validada por un grupo de expertos y existiendo una relación entre los ítems de la misma y los objetivos de la presente investigación.

Para el cuestionario final se realizó un Análisis Factorial de Componentes Principales, donde se obtienen valores adecuados para el índice KMO de 0.831 como en el test de esfericidad de Barlett $(p>.00)$. Los índices obtenidos en la matriz de covarianzas, presentaron ajustes satisfactorios para el índice RMSEA $=0.071$, como en el GFI $=$ 0.81 . Así, se obtienen 2 factores que integran el cuestionario:

1- Motivación hacia el aprendizaje (11 ítems): en este factor se abordan 
cuestiones vinculadas a la implicación de alumno hacia las tareas y su propio seguimiento a lo largo del proceso.

2- Relación social entre los integrantes de la clase (10 ítems): se integran cuestiones relacionadas con el clima de trabajado generado en clase y la gestión de roles en el trabajo de grupo.

\subsubsection{Cualitativos}

La extracción de información cualitativa de la investigación se realizó a través de una entrevista semi-estructurada a los dos docentes participantes en la misma. El objetivo era explorar los pensamientos y las sensaciones de los entrevistados para obtener una perspectiva interna de la experiencia (Patton, 2002). En base a esta idea, se desarrolló un guión tomando como referencia los conocimientos previos de los investigadores y trabajos similares de investigación. Como la naturaleza de la entrevista era semiestructurada, los investigadores pudieron añadir nuevas preguntas en base a las respuestas de los entrevistados para convertir la entrevista en una conversación (Patton, 2002). Este formato abierto permite explorar nuevas áreas para producir datos más ricos (Smith \& Osborn, 2003). Atendiendo a las variables del estudio se preguntaron cuatro cuestiones principales a cada uno de los docentes al finalizar las asignaturas; 1) ¿Hasta qué punto es beneficioso la inclusión de las NNTT en el aula? 2) ¿Cuál es el fin de su inclusión como elemento de aprendizaje? 3) ¿Es transcendente que el docente tenga un dominio elevado de las mismas? 4) ¿Qué pautas metodológicas y evaluativas son necesarias tener en cuenta para su implantación?

Toda la información relativa a estas respuestas se ha agrupado en tres categorías: 1"Rol metodológico y evaluativo en su puesta en práctica", 2- "Necesidad de conocimiento exhaustivo por el docente", 3- "Utilidad de las mismas para el aprendizaje".

\subsection{Diseño y procedimiento}

Las cuatro asignaturas que componen la muestra tienen un carácter semestral. El estudio es de carácter retrospectivo, ya que el alumnado ha cumplimentado el cuestionario una vez ha finalizado las asignaturas y conoce su calificación. A continuación se expone el enfoque metodológico que los docentes han dado a las asignaturas en cada uno de los grupos:

- Grupo A uso del audioblog: se emplea esta herramienta como instrumento formativo en la asignatura. El alumno, dividido en grupos, y a partir de las temáticas abordadas en la asignatura, realizará sus propios archivos de audio en los que reflexionará, profundizará o ampliará determinados contenidos. Estos audios serán subidos al blog creado en la asignatura con el fin de que sean escuchados por todos los miembros de la clase. A partir de aquí se generarán debates estructurados que permitan el acceso a otros links, vídeos o imágenes de la Web. Se comparan los audios elaborados por los alumnos con podcast de la misma temática presentes en la web y que también serán insertados en el blog. 
- Grupo B no uso del audioblog: los alumnos también trabajan en grupos pero no utilizan el audioblog como medio de difusión de su trabajo ni como elemento para compartir la información. Se trabaja mediante exposiciones grupales fundamentadas en el uso de diapositivas. El temario normalmente suele proporcionarlo el docente y es a partir de ahí donde el alumno genera su trabajo. No existe un feedback regulado y formativo ni entre profesor y alumno ni entre compañeros.

Al finalizar la asignatura, los estudiantes respondieron de forma individual en el aula al cuestionario en una única sesión de una hora de duración. En todo momento se garantizó el anonimato para que las respuestas de los estudiantes fueran lo más sinceras posibles, al igual que se garantizó la confidencialidad en el tratamiento de los datos.

\subsection{Análisis empleado}

La metodología de la investigación ha sido mixta, empleándose tanto un análisis cuantitativo (descriptivo e inferencial) como cualitativo (entrevistas). Se emplea un pretest-postest, comprobando en qué medida varía la motivación del alumnado y la relación social de la clase tras haber cursado las asignaturas. Esta complementariedad en el tratamiento de los datos otorgará un visionado más global de los resultados obtenidos, así como una mayor comprensión de los mismos.

\subsubsection{Cuantitativo}

Se lleva a cabo un tratamiento descriptivo, (medias y DT) y uno inferencial (Anovas) para cada uno de los dos grupos. El tratamiento de los datos en el pretest-postes se hace a través de los factores de análisis, viendo si existen diferencias significativas en los factores entre grupos antes y después de haberse llevado a cabo la asignatura. En el ANOVA se observa si existen diferencias intragrupales para las tres variables independientes utilizadas.

\subsubsection{Cualitativo}

El análisis cualitativo se realizó a partir de la recogida estructurada de información mediante una entrevista a los docentes participantes en la investigación. Los datos extraídos se analizaron a través del análisis de la temática del contenido (Libarkin y Kurdziel, 2002) y la comparación constante entre los datos (Denzin y Lincoln, 1994). El análisis del contenido se centró en la búsqueda de patrones en el texto, codificando los extractos coincidentes con los patrones cruzados (Saldaña, 2009). Los temas que surgieron en el primer análisis independiente fueron examinados críticamente por todos los investigadores a través de un diálogo reflexivo. La confiabilidad fue apoyada a través de la retroalimentación continua y el análisis participativo por parte de los investigadores que revisaron y perfeccionaron las categorías emergentes, para que los resultados pudieran ser considerados confiables, creíbles y transferibles (Lincoln y Guba, 1985). El objetivo era utilizar la información obtenida para dotarla de una mayor comprensibilidad a través de la transferencia de los resultados. Las categorías que surgieron de los datos se presentan de forma explícita a través de la sección de 
resultados y con el apoyo de varios ejemplos de textos (Cohn, 1991).

Para recapitular, organizar y obtener la saturación de los datos a partir de las categorías generadas en las preguntas realizadas a los dos docentes se ha utilizado el programa de computación WEFT QDA. El acrónimo empleado para el docente que utilizó el audioblog es DAB (docente audioblof) y para el que no las utilizó es DNB (docente no blog).

\section{RESULTADOS}

\subsection{Análisis cuantitativo: descriptivo}

$$
\text { PRE-TEST }
$$

POST-TEST

N Media DT Var. Media DT Var.

\begin{tabular}{lccccccc}
\hline & \multicolumn{7}{c}{ Grupo uso Audioblog (a) } \\
\hline F.1. Motivación & 75 & 3,52 & .239 & .057 & 4,01 & .183 & .033 \\
\hline F.2. Relación social & 75 & 3,31 & .318 & .101 & $4,22^{\text {aa* }^{*}}$ & .193 & .037 \\
\hline & \multicolumn{7}{c}{ Grupo no uso Audioblog (b) } \\
\hline F.1. Motivación & 87 & 3,61 & .228 & .051 & 3,72 & .296 & .087 \\
\hline F.2. Relación social & 87 & 3,56 & .341 & .116 & $3,24^{\text {ba* }^{*}}$ & .188 & .035 \\
\hline
\end{tabular}

Tabla 2. Comparación de medias por factores para cada uno de los grupos en el pretest-postest (nivel de significación en las diferencias: * $\mathrm{p}<.05$ )

Nota: Los superíndices reflejan los grupos entre los que se encuentran las diferencias significativas a nivel .05

Se observa cómo en el pretest no existen diferencias significativas en ninguno de los factores entre grupos, alcanzándose incluso medias más elevadas en el grupo que no utilizó el audioblog (los profesores es la primera vez que dan clase a estos alumnos). Al finaliza la asignatura, las medias de los dos factores se incrementan en el grupo A, mientras que en el $B$ en el factor de relación social se reducen. Es este factor en el que se encuentran diferencias significativas en el grupo A entre el pretest-postest y entre los dos grupos al finalizar la asignatura.

\subsection{Análisis inferencial: ANOVA}

BONFERRONI Y POST HOC A partir del análisis factorial realizado y en relación a los ítems relacionados con el aprendizaje obtenido por el alumno, se ha creado la variable de escala denominada "aprendizaje generado en la asignatura". Para ello se realiza un Anova de una vía para grupos independientes con el fin de comprobar si existen diferencias estadísticamente significativas acerca del aprendizaje generado, 
atendiendo a las variables independientes de edad, experiencia previa en el trabajo en grupo y asiduidad en el uso de las NNTT. Además se realiza un post hoc indicando en qué grupos se encuentra esas diferencias. La variable de edad se categoriza en: 1"entre 20 y 22 ", 2- "entre 23 y 25 " y $3-$ "más de 25 ". La de experiencia previa en trabajo en grupo en: 1- "más de tres veces", 2- "entre una y tres veces", 3- "nunca". La de asiduidad en el uso de las NNTT en: 1- "a diario", 2- "más de tres veces por semana"

\begin{tabular}{cccc}
\hline APRENDIZAJE GENERADO ASIGNATURA & $F$ & $g /$ & $p$ \\
\hline Grupo uso Audiblog (a) & & & \\
\hline Edad & 102.21 & 1 & $\mathbf{. 0 1 1 *}$ \\
\hline Experiencia previa trabajo en grupo & 96.51 & 2 & .331 \\
\hline Asiduidad en uso de NNTT & 69.54 & 1 & .187 \\
\hline Grupo no uso Audioblog (b) & & & \\
\hline Edad & 104.13 & 1 & .252 \\
\hline Experiencia previa trabajo en grupo & 97.91 & 2 & .352 \\
\hline Asiduidad en uso de NNTT & 75.84 & 1 & $\mathbf{. 0 4 1 * *}$
\end{tabular}

Tabla 3. Resumen de Anova (Bonferroni) para cada una de las variables independientes analizadas en el postest (edad, experiencia previa en trabajo en grupo y asiduidad en uso de NNTT)

*p $<.05$ entre "20-22 años" (media 4,71) y "más de 25 años" (media 3,93)

*p< 05 entre "esporádicamente" (media 3,37) "a diario" (media 4,18)

En el grupo que ha utilizado el audioblog se encuentran diferencias significativas en la variable de la edad, siendo los alumnos más jóvenes los que consideran que su uso formativo permite un mayor aprendizaje de los contenidos abordados $(F(75)=102,21$, $\mathrm{p}=.011$ ). En el grupo que no ha utilizado el audioblog las diferencias se encuentran en la variable de la asiduidad en el uso de las NNTT fuera del aula. Los que las utilizan esporádicamente son los que más valoran el aprendizaje obtenido en el grupo b ( $F$ $(87)=75,84, p=.041)$. La variable de experiencia previa en trabajo en grupo en otras asignaturas no influye de manera significativa en ninguno de los dos grupos generados en el estudio.

\subsection{Análisis cualitativo}

De toda la información extraída de las respuestas en las entrevistas realizadas a los docentes participantes, y con el fin de conocer su percepción sobre el uso de las NNTT como elemento motivacional del alumnado hacia los contenidos, se generaron tres categorías fundamentales: 1-Utilidad de las mismas para el aprendizaje, 2- Necesidad de conocimiento exhaustivo por el docente, 3- Rol metodológico y evaluativo en su 
puesta en práctica.

Utilidad de las mismas para el aprendizaje: Se observa cómo los dos docentes no otorgan la misma importancia al uso de estas herramientas de aprendizaje, mostrando diferentes percepciones sobre la implantación de las mismas como eje regulador de las asignaturas.

"La herramienta del audioblog no es buena ni mala de por sí [...]. Lo verdaderamente interesante radica en conocer cuál es el uso funcional y formativo que puede tener el mismo en clase". "De todos modos hay que ser consciente de los beneficios que tiene implementar herramienta virtuales como esta, ya que le otorga una dimensión mayor al aprendizaje"(DAB).

"No me preocupa demasiado el uso de las NNTT en clase [...]". " Creo que bastante ya las utilizan los alumnos fuera del aula como para que nosotros insistamos más en ello" [...] "Además estamos en la Universidad, estas competencias digitales deberían tenerlas adquiridas, yo les enseño los contenidos, ellos que decidan cómo quieren presentarlos o compartirlos con los demás"(DNB).

Necesidad de conocimiento exhaustivo por el docente: El grado de relevancia que otorga el docente a su competencia y control de las NNTT se convierte en un factor determinante y decisivo para su propia decisión a la hora de usarlas.

"No creo que el hecho de tener un dominio mayor o menor de las NNTT deba ser un condicionante para no utilizarlas en el aula" [...] (DAB). "Es casi seguro que algunos alumnos tendrán un conocimiento mayor que nosotros en el uso, pero nuestro rol ha de ser cómo poder regular el aprendizaje a partir de estas herramientas".

"Si te soy sincero me cuesta trabajar con algo de lo que yo no domino, al final uno siempre utiliza aquello de lo que sabe [...]". "No obstante, insisto en que esto no es competencia nuestra y al final si el alumno quiera utilizar estas herramientas es un cosa más a mayores (DNB).

Rol metodológico y evaluativo en su puesta en práctica: Se aprecia cómo el uso de las NNTT se encuentra supeditado al enfoque metodológico y evaluativo que utiliza el profesor en la asignatura.

"Es fundamental que usemos estas herramientas con fines formativos [...]". "En este caso pretendo que con el audioblog se potencie la comunicación en el aula, el hecho de que el alumno comparta experiencias" [...] "El audioblog es solamente un medio, otro año utilizaré otro instrumento [...]"(DAB).

"Hay que utilizar herramientas que se vinculen a la calificación que podamos poner al alumno [...]". "Los chicos en este caso han utilizado el Power Point porque exponían sus trabajos en clase. Esto me da a mí la información suficiente para saber cómo han hecho las cosas"(DNB). 


\section{DISCUSIÓN}

Se ha reflejado cómo a pesar de que en el pretest no existían diferencias significativas entre los dos grupos en relación a los dos factores de análisis, después de llevar a cabo las asignaturas el grupo que utilizó el audioblog obtuvo un aumento significativo en la relación social entre los integrantes de la clase. Además en este factor también se encontraron diferencias entre los dos grupos. Por lo tanto, en el grupo que utilizó el audioblog se obtuvo un clima más positivo de aula y una mayor motivación hacia los contenidos impartidos, siendo la variable independiente de la edad en la que se encuentran diferencias significativas en relación al aprendizaje generado. Sin embargo, en el grupo que no se utilizó el audioblog como herramienta formativa las diferencias en cuanto al aprendizaje obtenido se encontraron en la variable de asiduidad en el uso de las NNTT fuera del aula. Por otra parte, los docentes tienen una percepción diferente sobre el uso de las NNTT como herramienta formativa, discrepando en el carácter metodológico y el rol que ha de desempeñar el profesorado en su puesta en práctica.

Llama la atención observar cómo en el pretest los valores medios de cada factor son incluso más bajos en el grupo A que en el B, lo que refleja la homogeneidad de las opiniones entre grupos. Como destacan Gan y Hattie (2014) en este tipo de estudios el hecho de que los alumnos no partan de unas ideas preconcebidas potencia el efecto en los resultados que pueda tener el plan de intervención puesto en práctica. En el caso de la presente investigación, los dos docentes participantes es la primera vez que imparten clase a estos alumnos, por lo que sus percepciones en cuanto a los dos factores de análisis se fundamentan en las experiencias vivenciadas en otras asignaturas. Sin embargo al finalizar las asignaturas las valoraciones que hace el alumno varían sustancialmente, produciéndose en el grupo que utilizó el audioblog un incremento en los dos factores.

Puede admitirse por lo tanto que el empleo de esta herramienta formativa se asocia a una motivación y clima de relación social positivo en el aula. En este sentido Durán (2010) indica que experiencias relacionadas con el uso interactivo de instrumentos como el blog favorecen la cohesión social entre los alumnos, debido fundamentalmente al hecho de que los alumnos han de trabajar simultáneamente en diversidad de contenidos. Además, el feedback en la información es constante y no se ciñe únicamente a los horarios de clase, lo que facilita la reflexión del alumno en sus intervenciones y la planificación y organización en la propuesta de material a sus compañeros (Alvarado, 2014). No obstante Meyer (2011) indica que todos los aspectos positivos de estas herramientas pueden convertirse en desventajosos si no se planifica un seguimiento lógico, estructurado y coherente en su puesta en práctica. Del mismo modo Román, Cardemil y Carrasco (2011) advierten del peligro que tiene emplear estas herramientas con el único fin de trabajar las NT (sin que exista un argumento metodológico y didáctico), ya que puede derivar en un uso rutinario por parte del alumno que no genere reflexión y que únicamente siga la "inercia" generada en las asignaturas que utilizan estas herramientas.

El aumento de la motivación alcanzada en este caso parece deberse a la novedad que 
supone para el alumno generar sus propios audios y subirlos a la plataforma, contrastando los subidos por el resto de compañeros y analizando el contenido de los mismos. Stark, Sachau \& Albertson (2012) indican en una experiencia llevada a cabo en el desarrollo de un programa de radio en el aula, que el efecto motivador en el estudiante en este tipo de propuestas se encuentra en el hecho de presentar sus producciones a sus compañeros, comprobando cómo su trabajo además de ser conocido en el aula deriva en interacciones con otros miembros de la comunidad educativa.

Llama la atención en el presente estudio observar cómo en el postest del grupo B se reducen los valores de relación social entre los discentes. Este hecho indica que el alumno percibe que la dinámica metodológica desarrollada en clase no favorece la socialización, utilizando un enfoque evaluativo orientado de manera individual. Sin embargo en este grupo se han utilizado exposiciones orales en grupo mediante la presentación de diapositivas, algo que parece no haber contribuido a este factor. En este sentido García-Ruíz, González-Fernández y Contreras (2014) reflejan cómo el hecho de demandar trabajos en grupo a los estudiantes (entendidos estos como personas que se juntan para realizar una tarea) sin que se utilicen metodologías que favorezcan la interacción de roles, el registro de las tareas y la responsabilidad entre sus integrantes, puede provocar desajustes en el nivel de trabajo que conlleven un clima de trabajo que no sea positivo. Por lo tanto es fundamental que cada alumno sepa cuáles son tanto sus funciones dentro del grupo como la del resto de compañeros, generando procesos regulares de autoevaluación dentro del grupo y de coevaluación entre iguales (Birjandi \& Hadidi, 2012). Por tanto al trabajo en grupo hay que darle más transcendencia que el mero hecho de que una serie de alumnos se junten para realizar una tarea $y / 0$ actividad, debiendo estar enfocados hacia proyectos que aborden contenidos más globales y si es posible interdisciplinares (Mansilla, 2005). Estas afirmaciones tienen relación directa con los resultados obtenidos en el presente estudio, ya que el grupo que utilizó la metodología abierta y participativa a través del audioblog obtuvo una percepción sobre el clima relacional generado en el aula significativamente más elevada que el grupo B.

Dentro de las variables independientes utilizadas en el estudio para cada uno de los grupos se observa cómo es la edad donde se encuentran diferencias significativas respecto al aprendizaje generado en la asignatura. Son los alumnos más jóvenes los que entienden que esta herramienta de aprendizaje ha servido para generar un aprendizaje más real y transferible a diversos ámbitos. Aguado, Arranz, Rubio y Marín (2011) indican que actualmente en el ámbito universitario, y sobre todo en la formación semipresencial u online, acontecen dos situaciones: a) utilización de una metodología y evaluación fundamentada en las NNTT y b) disparidad en la características del alumnado (edad, tipo de formación, situación personal y/o familiar...). En este sentido los alumnos de mayor edad, normalmente con titulaciones previas, presentan un mayor desconocimiento sobre el uso de herramientas virtuales, algo que dificulta su asimilación inicial y repercute en su percepción sobre el desarrollo de la asignatura (Park \& Kim, 2014). Este hecho contrasta con los alumnos más jóvenes, que mayores conocedores del uso de plataformas, redes sociales, blogs...tienen una mayor capacidad de adaptación para comprender y manejar este 
tipo de herramientas e instrumentos, debido en parte a que ya lo han trabajado en la etapa obligatoria (Canessa \& Pisani, 2013).

Sin embargo, dentro de grupo B, que utilizó la presentación de diapositivas (Power Point, Prezi o similares) para exponer trabajos grupales en clase, las diferencias se han encontrado en función a la variable del uso de las NNTT fuera del aula. Los alumnos que las utilizan a diario perciben un menor aprendizaje generado en la asignatura. Este hecho puede entenderse en que los estudiantes que tienen una mayor competencia digital y que están más acostumbrados al uso de herramientas digitales perciben una mayor limitación del aprendizaje cuando en una asignatura no se aborda su tratamiento (Valdebenito \& Duran, 2013). Por el contrario, y tal como se refleja en el presente estudio, los alumnos que reconocen la utilización de las NNTT de manera esporádica se sienten más cómodos y su percepción es más favorable cuando en las asignaturas estas herramientas no se implementan de manera continua (Lee, Tsai, Chai \& Koh, 2014).

A pesar de que en las 4 asignaturas del estudio se haya trabajado en grupo, en la variable de experiencia de trabajo en grupo en otras asignaturas no se han encontrado diferencias. Esto puede reflejar la disparidad de criterios existentes entre docentes a la hora de entender cómo regular los agrupamientos en el aula y delimitar pautas de organización internas entre sus integrantes. Sin embargo, otras experiencias en el ámbito educativo (Giese, 2013) indican que los alumnos que más acostumbrados están a la realización de trabajos grupales presentan uno niveles más elevados de regulación del trabajo y un mayor aprendizaje de las temáticas específicas que se han trabajado.

En relación a la parte cualitativa del estudio, la discrepancia entre las opiniones de los docentes son evidentes, ya no solamente en relación al uso de herramientas concretas, sino al rol que debe desempeñar tanto el docente como el discente en su puesta en práctica. El profesor que ha utilizado el audioblog admite que la importancia no se encuentra en la herramienta en sí, sino el rol formativo que se le otorgue a la misma. Por el contrario el profesor del grupo B establece la ausencia de necesidad de trabajar en la universidad las NNTT, ya que no es competencia propia del docente. En este sentido García-Valcárcel, Hernández y Recamán (2012) indican que a pesar de que el alumnado actualmente tenga un manejo de las NNTT elevado, no implica que desde el aula debamos de dejar de abordarlas desde un enfoque educativo. Respecto al nivel que ha de tener el docente para aplicarlas en clase, el profesor del grupo A indica que el nivel en su dominio no ha de suponer un condicionante, mientras que al del grupo B le cohíbe el hecho de no manejarlas con fluidez para aplicarlas en el aula. Lau \& Lee (2015) reflejan que la tendencia actualmente en el ámbito universitario se dirige hacia una "virtualización", lo que provoca que el profesor deba modificar estructuras de trabajo más tradicionales que se basen únicamente en la reproducción de clases magistrales. Respecto a la tercera categoría generada, se observa cómo el uso de las NNTT se encuentra relacionado con la metodología y evaluación que el docente aplica en su asignatura. El profesor del grupo A valora la implantación del audioblog con fines formativos, mientras que el del grupo B entiende el uso de los instrumentos con una orientación más calificadora. Según Canabal y Castro (2012) 
este es uno de los aspectos más relevantes que gira alrededor de la concepción de la enseñanza, ya que el hecho de evaluar ha de enfocarse bajo un criterio único de aprendizaje, independientemente si después eso se traduce o no en una calificación.

\section{CONCLUSIONES}

En relación al primer objetivo de la investigación, se ha comprobado cómo el grupo que participó en el audioblog obtuvo tanto una mayor motivación hacia los contenidos como un mejor clima de relación social en el aula, siendo este el factor en el que se produjo un mayor incremento tanto en relación al pretest como en el contraste entre grupos. En el grupo que no se utilizó el audioblog descendieron ligeramente los valores medios de cada factor.

Respecto al segundo objetivo, en el grupo en el que se utilizó el audiblog ha sido la variable de edad en la que se han encontrado diferencias en las percepciones sobre el aprendizaje generado, siendo los alumnos más jóvenes los que presentaban unos valores más elevados. En el grupo $\mathrm{B}$ las diferencias se encontraron en la variable de experiencia en el uso de las NNTT fuera del aula. En este caso los alumnos que menos utilizan las NNTT en el día a día muestran una mayor percepción sobre el aprendizaje. En la variable de experiencia en el trabajo en grupo a lo largo de la titulación no se encuentran diferencias.

Atendiendo al tercer objetivo de la investigación referido a la percepción docente, se ha comprobado cómo los dos profesores que han impartido las asignaturas tienen diferentes criterios sobre la utilidad que puede tener el uso de herramientas virtuales en el aula. Esta diversidad de opiniones se encuentra directamente relacionada con la metodología y la evaluación utilizada en la asignatura.

Consideramos que este estudio puede ser de gran utilidad para todos aquellos docentes cuya práctica educativa se vincula a la utilización de herramientas e instrumentos virtuales con carácter formativo. Del mismo modo, también es de interés para todos aquellos profesores que desarrollan metodologías abiertas y participativas en sus clases, valorando la percepción del alumno como un criterio fundamental en el desarrollo de la asignatura.

El artículo presenta algunas limitaciones. En primer lugar únicamente participan alumnos de una facultad, por lo que sería interesante contrastar las percepciones de estudiantes de otras universidades. Por otro lado los alumnos forman parte de diferentes titulaciones. Para futuras investigaciones podría valorarse la opinión de alumnos de primer y cuarto curso de la misma titulación, comprobando cuál es la competencia digital que ha adquirido el alumno al finalizar la carrera en relación a cuando la comenzó.

\section{REFERENCIAS}

Aguado, D.; Arranz, V.; Rubio, A.V. \& Marín, S. (2011). Evaluación de un programa 
blended-learning para el desarrollo de la competencia trabajar en equipo. Psicothema, 23(3), 356-361.

Alvarado, M.A. (2014). Retroalimentación en educación en línea: una estrategia para la construcción del conocimiento. RIED: revista iberoamericana de educación a distancia, 17(2), 59-73.

Brodahl, C.; Hadjerrouit, S. \& Hansen, N. (2011). Collaborative Writing with Web 2.0 Technologies: Education Students' Perceptions. Journal of Information Technology Education, 10, 72-103.

Birjandi, P. \& Hadidi, N. (2012). The Role of Self-, Peer and Teacher Assessment in Promoting Iranian EFL Learners' Writing Performance. Assessment \& Evaluation in Higher Education, 375), 513-533.

Canabal, C. \& Castro, B. (2012). La evaluación formativacla utopía de la Educación superior? Pulso: revista de educación, 35, 215-229.

Canessa, E. \& Pisani, A. (2013). High School Open On-Line Courses (HOOC): A Case Study from Italy. European Journal of Open, Distance and E-Learning, 16(1), 131140.

Castejón, F.J.; Santos, M. \& Palacios, A. (2013). Cuestionario sobre metodología y evaluación en formación inicial en Educación Física. Revista Internacional de Medicina y Ciencias de la Actividad Física y el Deporte, 12(1), 1-23.

Cohn, P. J. (1991). An exploratory study on peak performance in golf. The Sport Psychologist, 5, 1-14.

Corbetta, P. (2007). Metodologías y técnicas de investigación social. Madrid: McGrawHill.

Davis, K. (2011). A Life in Bits and Bytes: A Portrait of a College Student and Her Life with Digital Media. Teachers College Record, 113(9), 1960-1982.

Denzin, N. K., y Lincoln, Y. S. (1994). Handbook of Qualitative Research. Thousand Oaks, CA: Sage.

Durán, J.F. (2010). La utilización del edublog en las aulas como dinamizador del proceso de enseñanza-aprendizaje. Docencia e Investigación: revista de la Escuela Universitaria de Magisterio de Toledo, 35(20), 205-243.

Gan, M. \& Hattie, J. (2014). Prompting Secondary Students' Use of Criteria, Feedback Specificity and Feedback Levels during an Investigative Task. Instructional Science: An International Journal of the Learning Sciences, 42(6), 861-878.

García-Valcárcel, A.G.; Hernández, A. \& Recamán, A. (2012). La metodología del aprendizaje colaborativo a través de las TIC: una aproximación a las opiniones de profesores y alumnos. Revista Complutense de Educación, 23(1), 161-188.

García-Ruíz, R.; González-Fernández, N. \& Contreras, P. (2014). La formación en competencias en la universidad a través de proyectos de trabajo y herramientas 2.0. Análisis de una experiencia. RUSC. Universities and Knowledge Society Journal, 
$11(1), 61-75$.

Gerich, D. (2013). Beyond the Class Blog: Creative and Practical Uses of Blogger for the ESL Classroom. TESOL Journal, 4(1), 175-181.

Giese, M. (2013). Moving from the Classroom to the Workplace: A Service-Learning Case Study of a Media Production Capstone Course. Metropolitan Universities, 24(1), 11-24.

Lau, K.; \& Lee, P.Y. (2015). The Use of Virtual Reality for Creating Unusual Environmental Stimulation to Motivate Students to Explore Creative Ideas. Interactive Learning Environments, 23(1), 3-18.

Lee, K.; Tsai, P.S.; Chai, C.S. \& Koh, J.H. (2014). Students' Perceptions of SelfDirected Learning and Collaborative Learning with and without Technology. Journal of Computer Assisted Learning, 30(5), 425-437.

Leemen, L. (2013). The Impact of Teachers' and Supervisors' Involvement on Students' Skill and Affective Development in Service-Learning. Educational Research and Reviews, 8(21), 1979-1987.

Libarkin, J. C. \& Kurdziel. J. P. (202). Research methodologies in science education: Qualitative data. Journal of Geoscience Education, 50, 195-200.

Lincoln, Y. S. \& Guba, E. (1985). Naturalistic inquiry. Newbury Park, CA: Sage.

Mansilla, V. (2005). Assessing Student Work at Disciplinary Crossroads. Change, 371), 14-17.

Martínez, F. (2013). Los trabajos tutorizados como método de aprendizaje a través de la investigación y la aplicación de las nuevas tecnologías. Ariadna: cultura, educación y tecnología, 1(1), 53-57.

Meneses, E. \& Llorente, M. (2010). Incorporación de nuevas estrategias de enseñanza en la Universidad: blogs en "Didáctica General". Educatio siglo XXI: Revista de la Facultad de Educación, 28(1), 191-208.

Meyer, K. (2011). Is Online Learning a Disruptive Innovation? Planning for Higher Education, 39(4), 44-53.

Nortes, R. \& Nortes-Checa, A. (2012). Enseñanza, aprendizaje y evaluación en el Grado de Maestro de Primaria. Educatio siglo XXI: Revista de la Facultad de Educaci, 30(2), 289-312.

Patton, M. Q. (2002). Qualitative Research and Evaluation Methods. Thousand Oaks, CA: Sage.

Park, S. \& Kim, C. (2014). Virtual Tutee System: A Potential Tool for Enhancing Academic Reading Engagement. Educational Technology Research and Development, 62(1), 71-97.

Patrut, B.; Patrut, M. \& Cmeciu, C. (2013). Social Media and the New Academic Environment: Pedagogical Challenges, 12(1), 512-516.

Ryan, M. (2013). The Pedagogical Balancing Act: Teaching Reflection in Higher 
Education. Teaching in Higher Education, 18(2), 144-145.

Saéz-López, J.M. (2013). La práctica pedagógica de las tecnologías de la información y la comunicación y su relación con los enfoques constructivistas. REICE: Revista Electrónica Iberoamericana sobre Calidad, Eficacia y Cambio en Educación, 10(1), 58-73.

Saldaña, J. (2009). The Coding Manual for Qualitative Researchers. Thousand Oaks, CA: Sage.

Salmerón, H.; Rodríguez, S. \& Gutiérrez, C. (2011). Metodologías que optimizan la comunicación en entornos de aprendizaje virtual. Comunicar: Revista científica iberoamericana de comunicación y educación, 34, 163-171.

Tan, Y.; \& Tan, S. (2010). A Metacognitive Approach to Enhancing Chinese Language Speaking Skills with Audioblogs. Australasian Journal of Educational Technology, 26(7), 1075-1089

Smith, J. A. \& Osborne, M. (2003). Interpretative Phenomenological Analysis. In J.A. Smith (Ed.) Qualitative psychology: A practical guide to research methods. (2nd ed., pp. 53-79). London: Sage.

Stark, E.; Sachau, D. \& Albertson, D. (2012). Producing a Radio Show about Psychological Science: The Story of "Psychological Frontiers". Teaching of Psychology, 39(1), 42-44.

Valdebenito, V.; \& Duran, D. (2013). La tutoría entre iguales como un potente recurso de aprendizaje entre alumnos: Efectos de la fluidez y comprensión lectora. Perspectiva Educacional, 42(2), 154-176.

Vera, J.A. (2010). Dilemas en la negociación del currículo con el alumnado a partir de la cesión de responsabilidad de la evaluación en el aula de Educación Física. Revista de investigación en educación, 7, 72-82.

Wang, C.H.; Ke, Y.T.; Wu, J.T. \& Hsu, W.H. (2012). Collaborative Action Research on Technology Integration for Science Learning. Journal of Science Education and Technology, 21(1), 125-132.

White, R. (2007). Balance in Assessment. Measurement: Interdisciplinary Research and Perspectives, 5(1), 65-67.

\section{AUTOR/ES:}

\section{David Hortigüela Alcalá}

Doctor Internacional en Educación. Máster en Educación y Sociedades Inclusivas. Licenciado en Ciencias de la Actividad Física y del Deporte. Maestro Especialista en Educación Física. Graduado en Primaria mención en inglés. Posgrado en Bilingüismo. Docente en el Área de Expresión Corporal del Departamento de Didácticas Específicas. Autor de diversidad de artículos publicados en revistas científicas, libros, capítulos de 
libros y ponente en variedad de congresos nacionales e internacionales. Colaborador en varios proyectos I+D+I y de innovación docente. Revisor de diferentes revistas en el ámbito científico. Especialista en líneas de investigación vinculadas a la evaluación formativa, metodología y didáctica de la educación física, innovación en el aula y ciclos de investigación-acción.

\section{David Hortigüela Alcalá}

Doctor en Ciencias de la Actividad y del Deporte. Maestro Especialista en Educación Física. Docente en el Área de Educación Física y Deportiva del CAFD de León. Autor de diversidad de artículos publicados en revistas científicas, libros, capítulos de libros y ponente en variedad de congresos nacionales e internacionales. Colaborador en varios proyectos I+D+I y de innovación docente. Revisor de diferentes revistas en el ámbito científico. Especialista en líneas de investigación vinculadas a la programación, trabajo de las competencias clave, aplicación de metodologías activas y participativas en el aula y de métodos cualitativos aplicados en el marco de investigación. 